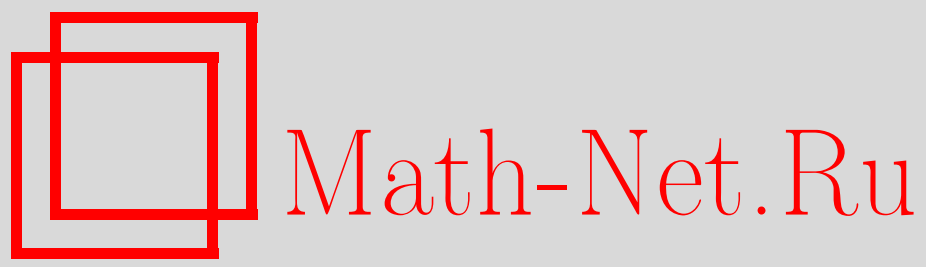

В. А. Кайманович, А. Г. Эршлер, Непрерывность асимптотических характеристик для случайных блужданий на гиперболических группах, Функи. анализ и его прил., 2013, том 47, выпуск 2, 84-89

DOI: https://doi.org/10.4213/faa3110

Использование Общероссийского математического портала MathNet.Ru подразумевает, что вы прочитали и согласны с пользовательским соглашением http://www . mathnet.ru/rus/agreement

Параметры загрузки:

IP : 3.85 .5 .30

26 апреля 2023 г., $17: 12: 29$

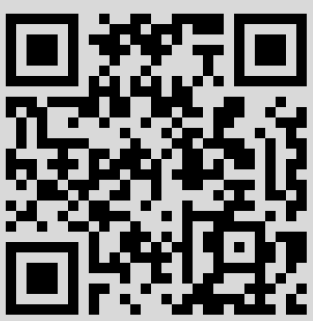


(1970), 273-303. [5] W. B. Johnson, G. Schechtman, Ann. Probab., 17:2 (1989), 789808. [6] С. В. Асташкин, Ф. А. Сукочев, Зап. научн. семин. ПОМИ, 345 (2007), 25-50. [7] С. В. Асташкин, Ф. А. Сукочев, УМН, 65:6 (2010), 3-86. [8] M. Sh. Braverman, Independent Random Variables and Rearrangement Invariant Spaces, Cambridge Univ. Press, Cambridge, 1994. [9] В. М. Круглов, ТВП, 15:2 (1970), 331-336. [10] С. Г. Крейн, Ю. И. Петунин, Е. М. Семенов, Интерполяция линейных операторов, Наука, М., 1978. [11] C. Bennett, R. Sharpley, Interpolation of Operators, Academic Press, Boston, MA, 1988. [12] В. А. Родин, Е. М. Семенов, Функц. анализ и его прил., 13:2 (1979), 91-92. [13] F. Albiac, N. J. Kalton, Topics in Banach Space Theory, Graduate Texts in Math., vol. 233, Springer-Verlag, New York, 2006. [14] J. Creekmore, Nederl. Akad. Wetensch. Indag. Math., 43:2 (1981), 145-152. [15] S. J. Dilworth, in: Handbook of the geometry of Banach spaces, vol. 1, North-Holland, Amsterdam, 2001, 497-532. [16] L. E. Dor, T. Starbird, Compositio Math., 39:2 (1979), 141-175. [17] L. E. Dor, Ann. of Math., 102:3 (1975), 463-474. [18] F. L. Hernandez, E. M. Semenov, J. Funct. Anal., 169:1 (1999), 52-80.

Самарский госуниверситет

e-mail: astashkn@ssu.samara.ru

Поступило в редакцию 10 октября 2011 г.

\title{
Непрерывность асимптотических характеристик для случайных блужданий на гиперболических группах
}

\author{
(c) 2013. В. А. КАЙМАНОВИЧ, А. Г. ЭРШЛЕР
}

Имеется целый ряд естественных численных инвариантов, связанных со случайным блужданием на счетной группе $G$, задаваемым вероятностной мерой $\mu$. В частности, это норма переходного оператора, его спектральный радиус, показатель экспоненциального убывания вероятностей возвращения, скорость ухода на бесконечность, асимптотическая энтропия. Близким родственником скорости ухода на бесконечность также является спектр показателей Ляпунова, определенный для линейных групп.

Для отдельно взятого случайного блуждания $(G, \mu)$ эти инварианты и взаимоотношения между ними были предметом изучения, начиная с основополагающей работы Кестена, вышедшей еще в 1959 г. Тем не менее вопрос об их зависимости от переходной меры $\mu$ на одной и той же группе $G$ исследован в куда меньшей степени.

Мы описываем новый метод, основанный на использовании условных случайных блужданий и позволяющий устанавливать непрерывность асимптотической энтропии как функции переходной меры при достаточно слабых предположениях о мерах (конечность первого момента). Он сводится к проверке равномерности в ленточном критерии описания границы Пуассона.

Несмотря на то, что в настоящей заметке мы ограничиваемся рассмотрением словарно гиперболических групп, наш подход также применим и в ряде других

* Работа над этой заметкой осуществлялась при поддержке European Research Council в рамках European Union Seventh Framework Programme (FP7/2007-2013), ERC grant agreement 257110-RAWG; ANR (France) в рамках проекта "DiscGroup: facettes des groupes discrets"; программы Canada Research Chairs; NSERC (Canada). 
случаев, когда граница Пуассона идентифицируется с подходящей геометрической границей (в частности, для групп, действующих на гиперболических пространствах, дискретных подгрупп полупростых групп Ли и т.д.). Более того, этот метод может быть использован и для тех обобщений обычных случайных блужданий, на которые распространяется ленточный критерий. Их пространства состояний могут быть как дискретными (например, для случайных блужданий в случайной среде или по отношениям эквивалентности), так и непрерывными (например, для броуновского движения на накрывающих многообразиях или на слоениях). Мы вернемся к этому вопросу в более подробной публикации.

1. Aсимптотическая энтропия случайного блуждания на счетной группе $G$, заданного вероятностной мерой $\mu$, определяется как предел

$$
h(\mu)=\lim _{n} \frac{H\left(\mu^{* n}\right)}{n}
$$

нормированных энтропий $n$-кратных сверток меры $\mu$ [5]. Она играет важную роль в теории случайных блужданий, в частности, $h(\mu)=0$ тогда и только тогда, когда граница Пуассона случайного блуждания тривиальна ([6], [13]).

В некоторых случаях асимптотическая энтропия может быть вычислена явно, например, если переходная мера сосредоточена на множестве свободных образующих в свободной группе [14] или в свободном произведении [18] (с использованием формулы для $h(\mu)$ в терминах гармонической меры на границе Пуассона [13]), а также для некоторых случайных блужданий на лампочных группах [7].

На множестве вероятностных мер на счетной группе $G$ имеется естественная топология поточечной сходимости:

$$
\mu_{i} \rightarrow \mu \Longleftrightarrow \mu_{i}(g) \rightarrow \mu(g) \quad \forall g \in G .
$$

Как нетрудно видеть, сходимость в этой топологии равносильна сходимости по вариации:

$$
\mu_{i} \rightarrow \mu \Longleftrightarrow\left\|\mu_{i}-\mu\right\|=\sum_{g}\left|\mu_{i}(g)-\mu(g)\right| \rightarrow 0 .
$$

Поскольку в определении $h(\mu)$ участвует субаддитивная последовательность энтропий сверток $H\left(\mu^{* n}\right)$, асимптотическая энтропия полунепрерывна сверху как функция переходной меры $\mu$ [4]. В упомянутых выше примерах явного вычисления асимптотической энтропии ее зависимость от $\mu$ является непрерывной. Она также непрерывна для мер, сосредоточенных на конечном подмножестве лампочной группы над $\mathbb{Z}[7]$.

Тем не менее есть и целый ряд примеров, показывающих, что, вообще говоря, асимптотическая энтропия не является непрерывной. Так, существуют меры $\mu$ на локально конечных группах с $h(\mu)>0$, хотя для всякой меры с конечным носителем на такой группе асимптотическая энтропия очевидным образом равна 0 ([1], [13]).

В классе конечно порожденных групп подобного рода примеры доставляют лампочные группы. Дело в том, что имеется естественное семейство случайных блужданий на этих группах, для которых граница Пуассона тривиальна (т. е. $h(\mu)=0)$ тогда и только тогда, когда факторблуждание на базе такой группы возвратно [13], в то время как множество мер с этим последним свойством не обязано быть замкнутым (это так, например, для двумерной целочисленной решетки $\mathbb{Z}^{2}$ или для бесконечной диэдральной группы $\left.\mathbb{D}_{\infty}\right)$. Группа $\mathbb{D}_{\infty}$ также 
дает пример разрывности в случае, когда переходные меры сосредоточены на одном и том же конечном множестве [7]. Тем не менее мы не знаем ни одного подобного примера, в котором рассматриваемые переходные меры были бы симметричны и имели один и тот же конечный носитель.

Многие другие проблемы, связанные с зависимостью асимптотической энтропии от переходной меры и ее носителя, обсуждаются в [3], [7].

2. Отправной точкой нашего подхода является то обстоятельство, что разность

$$
\Delta_{n}(\mu)=H\left(\mu^{* n}\right)-n h(\mu)
$$

можно интерпретировать как математическое ожидание энтропий $H\left(\mu_{\gamma}^{n}\right)$ распределений в момент времени $n$ условных случайных блужданий, заданных точками $\gamma$ границы Пуассона [2]. Если функции $\mu \mapsto H\left(\mu^{* n}\right)$ непрерывны (например, это так, если меры $\mu$ удовлетворяют равномерному условию конечности первого момента - см. ниже), то задача о непрерывности асимптотической энтропии сводится к доказательству того, что сходимость $\Delta_{n}(\mu) / n \rightarrow 0$ равномерна по $\mu$. Приведенное выше соотношение было впервые установлено в контексте идентификации границы Пуассона, и впоследствии оно использовалось в «ленточном критерии» (strip criterion) из [11], [12]. Мы вводим равномерную версию этого критерия, позволяющую нам доказать непрерывность асимптотической энтропии.

Пусть $\delta(g)=d(e, g)$ - «норма» на группе $G$, заданная левоинвариантной метрикой $d$ конечного экспоненциального роста (например, длина слов, определенная некоторым конечным множеством образующих). Первым моментом меры $\mu$ на $G$ относительно нормы $\delta$ называется полная масса меры $\delta \mu$ :

$$
|\mu|=|\mu|_{\delta}=\|\delta \mu\|=\sum_{g \in G} \delta(g) \mu(g) .
$$

Будем говорить, что семейство $\mathscr{M}$ мер на $G$ имеет равномерный первый момент, если семейство соответствующих мер $\{\delta \mu\}_{\mu \in \mathscr{M}}$ является плотным (tight), T. e.

$$
\delta \mu\left(G \backslash K_{n}\right)=\sum_{g \in G \backslash K_{n}} \delta(g) \mu(g) \rightarrow 0 \quad \text { равномерно по } \mu \in \mathscr{M}
$$

для любой исчерпывающей последовательности конечных множеств $K_{n}$.

Для задания другого условия на семейство $\mathscr{M}$ нам понадобится пара борелевских $G$-пространств $\widehat{B}, B$, таких, что существуют $G$-инвариантное подмножество $\Lambda \subset \widehat{B} \times B$ и $G$-эквивариантное отображение $S$, сопоставляющее всякой паре точек $(\hat{b}, b) \in \Lambda$ собственное подмножество («ленту») в $G$. Ленты, заданные отображением $S$, имеют равномерно субэкспоненииальный рост, если

$$
\frac{1}{n} \log \left|B_{n} \cap S(\hat{b}, b)\right| \rightarrow 0 \quad \text { равномерно по }(\hat{b}, b) \in \Lambda,
$$

где $B_{n}$ обозначает шар радиуса $n$ метрики $d$ с центром в единице группы.

Обозначим через

$$
\Lambda_{R}=\{(\hat{b}, b) \in \Lambda: d(e, S(\hat{b}, b)) \leqslant R\}
$$

исчерпание множества $\Lambda$ (при $R \rightarrow \infty)$, определенное окрестностями лент. Если для всякой меры $\mu \in \mathscr{M}$ пространство $B$ (соответственно $\widehat{B}$ ) снабжено вероятностной мерой $\nu$ (соответственно $\hat{\nu})$, делающей его $\mu$-границей (соответственно 
$\hat{\mu}$-границей) (здесь $\hat{\mu}(g)=\mu\left(g^{-1}\right)$ обозначает обращенную меру), и

$$
\hat{\nu} \otimes \nu\left(\Lambda_{R}\right) \rightarrow 1 \text { равномерно по } \mu \in \mathscr{M},
$$

то мы будем говорить, что семейство $\mathscr{M}$ удовлетворяет равномерному ленточному критерию. [Заметим, что в силу обычного ленточного критерия, примененного к индивидуальным мерам $\mu \in \mathscr{M}$, фигурирующие в этом условии $\mu$ - и $\hat{\mu}$-границы в действительности совпадают с соответствующими границами Пуассона.]

Теорема 1. Если семейство $\mathscr{M}$ вероятностных мер на группе $G$ и ленточное отображсение $S$ удовлетворяют равномерным условиям (M), (G) и (S), то асимптотическая энтропия непрерьвна на $\mathscr{M}$.

Доказательство сводится к равномерной оценке энтропий $H\left(\mu_{\gamma}^{n}\right)$ условных случайных блужданий, осуществляемой путем разбиения группы на три подмножества $G_{i}$ с последующей оценкой энтропии на каждом из этих подмножеств: $G_{1}$ - это пересечение шара радиуса $n L$ относительно нормы $\delta$ с $R$-окрестностью соответствующей ленты, $G_{2}$ - оставшаяся часть этого шара, а $G_{3}$ - дополнение шара в $G$. Константы $R$ и $L$ выбираются здесь при помощи условий $(\mathrm{S})$ и $(\mathrm{M})$ соответственно с таким расчетом, чтобы мера подмножества $G_{1}$ была близка к 1 равномерно по $\mu$.

3. В случае словарно гиперболических групn (по поводу их определения и основных свойств мы отсылаем читателя к первоначальной работе Громова [10], а также к изложению в [9]) из теоремы 1 следует

Теорема 2. Если $\mu_{i}$ - последовательность вероятностных мер на словарно гиперболической группе $G$ с равномерным первым моментом, сходящаяся $\kappa$ предельной вероятностной мере $\mu$, то $h\left(\mu_{i}\right) \rightarrow h(\mu)$.

Равномерность первого момента $(\mathrm{M})$ в этой формулировке понимается относительно длины слов, заданной некоторым конечным множеством образующих. Поскольку все подобные функции длины на конечно порожденной группе эквивалентны, условие $(\mathrm{M})$ (равно как и условия $(\mathrm{G})$ и $(\mathrm{S})$ ) не зависит от выбора конкретной системы образующих.

Напомним, что подгруппа $G^{\prime}$ словарно гиперболической группы $G$ называется элементарной, если она или конечна, или является конечным расширением группы $\mathbb{Z}$. Это равносильно тому, что для действия $G^{\prime}$ на гиперболической границе $\partial G$ существует конечное инвариантное подмножество [10, 8.2].

Обозначим через $\operatorname{gr}(\mu)$ группу, порожденную носителем меры $\mu$. Если $\operatorname{gr}(\mu)$ - элементарная подгруппа группы $G$, то $h(\mu)=0$ и утверждение теоремы 2 следует из верхней полунепрерывности асимптотической энтропии.

В противном случае, если подгруппа $\operatorname{gr}(\mu)$ неэлементарна, то траектории случайного блуждания группы $(G, \mu)$ сходятся на бесконечности к гиперболической границе $\partial G$ [12]. Возникающее при этом распределение точек выхода на границу называется гармонической мерой. Поскольку $\mu_{i} \rightarrow \mu$, подгруппы $\operatorname{gr}\left(\mu_{i}\right)$ также неэлементарны для всех достаточно больших $i$ и, следовательно, соответствующие гармонические меры тоже определены корректно. Таким образом, в ленточном критерии можно положить $\widehat{B}=B=\partial G$, а в качестве $\Lambda$ взять дополнение диагонали в $\partial B \times \partial B$. Лента $S(\hat{b}, b)$ определяется тогда как объединение всех геодезических (относительной фиксированной метрики слов), соединяющих граничные точки $\hat{b} \neq b \in \partial G$, так что условие (G) очевидным образом выполнено [12]. Наконец, поскольку множество $\Lambda$ открыто в $\partial B \times \partial B$, 
условие $(\mathrm{S})$ вытекает из приведенной ниже леммы 3 (Ив Гуиварх обратил наше внимание на то, что эта лемма - простое следствие того известного факта, что в неэлементарном случае гармоническая мера блуждания $(G, \mu)$ является единственной $\mu$-стационарной мерой на гиперболической границе).

Лемма 3 (непрерывность гармонических мер). Пусть $\mu_{i}-$ последовательность вероятностных мер на словарно гиперболической группе $G$, сходящаяся $\kappa$ предельной вероятностной мере $\mu$, для которой группа $\operatorname{gr}(\mu)$ неэлементарна. Тогда последовательность гармонических мер блужданий $\left(G, \mu_{i}\right)$ слабо сходится к гармонической мере блужсания $(G, \mu)$.

Замечание 4. Что касается дальнейших свойств асимптотической энтропии как функции переходной меры $\mu$ на словарно гиперболической группе, то было бы интересно узнать, является ли зависимость $h(\mu)$ от значений $\mu(g), g \in K$, аналитической для мер с конечным носителем $K$. Для свободных групп положительный ответ на этот вопрос был с использованием термодинамического формализма дан в [16].

4. Напомним, что скорость ухода на бесконечность (часто также называемая сносом) случайного блуждания $(G, \mu)$ относительно нормы $\delta$ определяется как

$$
\ell(\mu)=\lim _{n} \frac{\left|\mu^{* n}\right|_{\delta}}{n} .
$$

Наряду с использованием для доказательства непрерывности асимптотической энтропии, лемма 3 может быть использована и для доказательства непрерывности скорости ухода на бесконечность для словарно гиперболических групп:

Теорема 5. Пусть $\mu_{i}$ - последовательность вероятностных мер с равномерным первым моментом на словарно гиперболической группе $G$, сходящаяся $\kappa$ предельной вероятностной мере $\mu$, для которой группа $\operatorname{gr}(\mu)$ неэлементарна. Тогда $\ell\left(\mu_{i}\right) \rightarrow \ell(\mu)$.

Доказательство теоремы 5 основывается на «квазификации» восходящей к Фюрстенбергу [8] формулы для скорости ухода на бесконечность как интеграла от коцикла Буземана по гармонической мере на границе.

Замечание 6. Для асимптотической энтропии существует похожая формула с участием интеграла от коцикла Радона-Никодима гармонической меры [13]. Тем не менее зависимость коцикла Радона-Никодима от переходной меры $\mu$ делает использование этой формулы для анализа функции $\mu \mapsto h(\mu)$ проблематичным.

Замечание 7 (добавлено после принятия статьи к публикации). Развивая свой подход из [16], Ледраппье в недавней работе [15] доказал, что для мер с конечным носителем на словарно гиперболических группах зависимость асимптотической энтропии от меры липшицева. Используя иной подход, Матье [17] установил, что при дополнительном предположении симметричности рассматриваемых мер эта зависимость в действительности дифференцируема, и интерпретировал ее производную.

\section{ЛитЕРАТУРА}

[1] В. А. Кайманович, Зап. научн. сем. ЛОМИ, 123 (1983), 167-184. [2] В. А. Кайманович, Докл. АН СССР, 280:5 (1985), 1051-1054. [3] А. М. Вершик, YMH, 55:4(334) (2000), 59-128. [4] G. Amir, O. Angel, B. Virág, http://arxiv.org/abs/ 0905.2007. [5] A. Avez, C. R. Acad. Sci. Paris Sér. A-B, 275 (1972), A1363-A1366. 
[6] Y. Derriennic, in: Conference on Random Walks (Kleebach, 1979), Astérisque, vol. 74, Soc. Math. France, Paris, 1980, 183-201, 4. [7] A. Erschler, in: Random walks, boundaries and spectra. Proceedings of the workshop on boundaries, Graz, Austria, June 29-July 3, 2009 and the Alp-workshop, Sankt Kathrein, Austria, July 4-5, 2009, Progress in Probability, vol. 64 (eds. Lenz, Daniel et al.), Birkhäuser, Basel, 2011, 55-64. [8] H. Furstenberg, Trans. Amer. Math. Soc., 108 (1963), 377-428. [9] Sur les groupes hyperboliques d'après Mikhael Gromov, Papers from the Swiss Seminar on Hyperbolic Groups held in Bern, 1988, Progress in Mathematics, vol. 83 (eds. É. Ghys, P. de la Harpe), Birkhäuser, Boston, MA, 1990. [10] M. Gromov, Hyperbolic groups, in: Essays in group theory, Math. Sci. Res. Inst. Publ., vol. 8, Springer-Verlag, New York, 1987, 75-263. [11] V. A. Kaimanovich, C. R. Acad. Sci. Paris Sér. I Math., 318:1 (1994), 59-64. [12] V. A. Kaimanovich, Ann. of Math. (2), 152:3 (2000), 659-692. [13] V. A. Kaimanovich, A. M. Vershik, Ann. Probab., 11:3 (1983), 457-490. [14] F. Ledrappier, in: Topics in probability and Lie groups: boundary theory, CRM Proc. Lecture Notes, vol. 28, Amer. Math. Soc., Providence, RI, 2001, 117152. [15] F. Ledrappier, http://arxiv.org/abs/1110.3156. [16] F. Ledrappier, Groups Geom. Dyn., 6:2 (2012), 317-333. [17] P. Mathieu, http://arxiv.org/abs/1209.2020. [18] J. Mairesse, F. Mathéus, J. Lond. Math. Soc. (2), 75:1 (2007), 47-66.

Department of Mathematics and Statistics,

University of Ottawa

Поступило в редакцию

e-mail: vkaimano@uottawa.ca

2 июля 2012 г.

C.N.R.S., Département de Mathématiques,

Université Paris Sud, Orsay, France

e-mail: anna.erschler@math.u-psud.fr

УДК 517.982

\section{Пространства гладких функций, порожденные неоднородными дифференциальными выражениями*}

\section{(c) 2013. С. В. Кисляков, Д. В. Максимов, Д. М. Столяров}

Пространства непрерывно дифференцируемых функций на многообразиях размерности по крайней мере 2 (здесь мы ограничимся торами) в невырожденных случаях не изоморфны пространству $C(K)$ ни для какого компакта $K$ (по теореме Милютина то же самое можно выразить, сказав «не изоморфны пространству $C[0,1] »)$. Смысл этого высказывания уточнялся постепенно, история вопроса была подробно изложена в [3]. Мы обратимся сразу к общей ситуации, описанной в [3]. Пусть $T=\left(T_{1}, \ldots, T_{l}\right)$ - набор дифференциальных операторов с постоянными коэффициентами на торе $\mathbb{T}^{n}$. (Частная производная $\partial_{k}$ на торе - это оператор $g \mapsto \frac{\partial}{\partial t} g\left(e^{2 \pi i t}\right), t \in \mathbb{R}$, применяемый по $k$-й переменной.) На множестве тригонометрических полиномов от $n$ переменных введем полунорму $\|f\|_{T}=\max _{1 \leqslant j \leqslant l}\left\|T_{j} f\right\|_{C\left(\mathbb{T}^{n}\right)}$. После факторизации по ядру и пополнения получаем пространство $C^{T}\left(\mathbb{T}^{n}\right)$.

Если набор $T$ состоит из всех дифференциальных мономов порядка не выше $s$, возникает обычное пространство $C^{(s)}\left(\mathbb{T}^{n}\right)$, которое действительно не вкладывается дополняемо в $C[0,1]$ при $n \geqslant 2$ и $s \geqslant 1$, см., например, [2]. (Умест-

*Работа выполнена при поддержке Лаборатории Чебышева СПБГУ (грант Правительства РФ, дог. 11.G34.31.0026) и РФФИ (грант 11-01-00526). 